\title{
Electromagnetic Interference between External Defibrillator and Cardiac Resynchronization Therapy-Pacemaker (CRT-P) Devices
}

\author{
HAMID GHANBARI, M.D., * HAZIM AL-AMERI, M.D.,† JESSICA OTTINO, R.N., B.S.N.,† \\ CORDELL HASTINGS, R.N.,† JAMES KIPPOLA, B.S.,‡ IONI GUERON, B.S.,‡ \\ MARCOS DACCARETT, M.D., + and CHRISTIAN MACHADO, M.D.† \\ From the *Division of Cardiology, University of Michigan, Ann Arbor, Michigan; †Division of Cardiology, \\ Providence Hospital and Medical Center, Southfield, Michigan; and ‡RF Management, Boston Scientific \\ Corporation, St. Paul, Minnesota
}

Background: Implantable heart rhythm devices are susceptible to interference in hospitals where electromagnetic interference (EMI) sources are ubiquitous.

Case Description: We report three cases in which EMI from the external defibrillator caused the inability to interrogate Boston Scientific cardiac resynchronization therapy-pacemaker (CRT-P) devices. We have documented interference with the Boston Scientific CRT-P Contact Renewal device model numbers H120/H125 (Natick, MA, USA) and two brands of external defibrillators: the Philips Heartstart XL model number M4735A (Andover, MA, USA) and the Hewlett-Packard Codemaster model number M1722B (Palo Alto, CA, USA). For device implants, we routinely place external pacing pads with the external defibrillator in the "standby" mode for transcutaneous pacing so that only the pacer "start/stop" button needs to be pressed when necessary. We have not been able to interrogate three consecutive Boston Scientific CRT-P devices prior to closure while the external defibrillator had the back-up pacing mode on "standby." In our initial case, a second device was opened because this interaction was not recognized. We documented EMI with the standby pacing mode ON and discovered that by disabling only the "standby" pacing mode on the external defibrillator, the device could be interrogated without difficulty.

Conclusions: This is a case series reporting EMI with a Boston Scientific CRT-P Contact Renewal device H120/H125 telemetry from an external defibrillator with pacing mode on "standby." Failure to recognize this important interaction may lead to inappropriate device and resource utilization. (PACE 2011; 34:1087-1091)

\section{CRT, electromagnetic interference, biomedical engineering}

\section{Introduction}

The short-range wanded telemetry function incorporated in implantable heart rhythm devices (used for programming and retrieving medical data) may be susceptible to interference in hospitals where potential electromagnetic interference (EMI) sources are ubiquitous. ${ }^{1}$ EMI may be radiated or conducted and may be present in many different forms, including radiofrequency waves, microwaves, ionizing radiation, acoustic radiation, static and pulsed radiation, static and pulsed magnetic fields, and electric currents. ${ }^{2} \mathrm{We}$ report three cases in which EMI from external defibrillators with the pacer mode in the "standby" position caused the inability for the programmer to communicate with the pacemaker.

\footnotetext{
Address for reprints: Christian Machado, M.D., Electrophysiology, Providence Hospital and Medical Center, 16001 W. Nine Mile Road, Southfield, MI 48075. Fax: 248-849-2480; e-mail: cmachado@comcast.net

Received July 25, 2010; revised March 20, 2011; accepted March 26, 2011.

doi: 10.1111/j.1540-8159.2011.03130.x
}

\section{Description}

In our institution, we routinely place external defibrillator pacing pads on patients receiving pacemakers. The external defibrillators are placed in the "standby" mode so that only the "start/stop" button needs to be pressed if rescue pacing is warranted. The Philips Heartstart XL, M4735A (Andover, MA, USA) and Hewlett-Packard Codemaster, M1722B (Palo Alto, CA, USA; hereafter referred to as the "defibrillator") are the external defibrillators available at our institution. Boston Scientific implantable heart rhythm devices are exclusively implanted at our institution. We report three cases in which cardiac resynchronization therapy pacemaker (CRT-P) devices were implanted, yet unable to communicate with the programmer due to EMI (Fig. 1). It was not until the pacer mode on the defibrillator was placed in the OFF position that the EMI disappeared and communication between the programmer and the pacemaker was established. The defibrillator prevented the Boston Scientific CRT-P Contact Renewal device (H120 and H125; Natick, MA, USA) from maintaining short-range (also known

(C)2011, The Authors. Journal compilation (C)2011 Wiley Periodicals, Inc. 


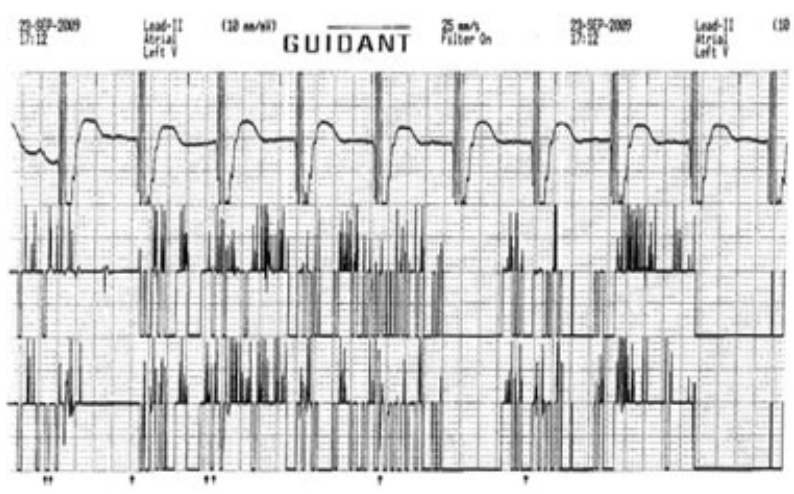

Figure 1. EMI on the programmer real-time wanded telemetry display with standby pacing mode enabled on external defibrillator.

as "wanded" telemetry) communication with its associated programmer (Fig. 2). In the first case, a new generator was opened to rule out device malfunction prior to determining the defibrillator as the cause of the interference. The interference with subsequent inability to communicate with the device was reproduced when the device was placed on the chest wall of a volunteer with defibrillator pads in place while the pacing was in the "standby" mode.

\section{Experiment}

We conducted an experiment in vitro to investigate the described interaction. We utilized a glass aquarium filled with $0.9 \%$ saline solution, the defibrillator, Boston Scientific model H120 CRT-P and Boston Scientific IS-1 bipolar leads (PG), Boston Scientific model 3120 programmer (PRM), and Tektronix model 754 Oscilloscope (Beaverton, OR, USA).

The saline solution is commonplace for performing in vitro evaluations of EMI as recom-

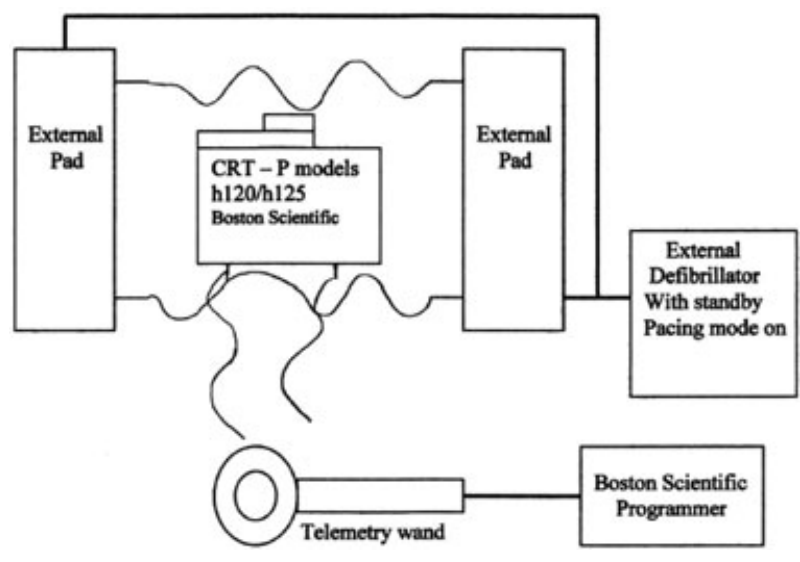

Figure 2. A schematic depicting the theorized coupling of EMI. mended by the Association for the Advancement of Medical Instrumentation (AAMI) PC69: 2007. ${ }^{3}$ The PG and defibrillator pads were submerged in the saline-filled tank with the PG positioned between the defibrillator pads. The pads were separated by a distance of approximately $30 \mathrm{~cm}$. Spurious emissions from the defibrillator cables and pads were measured using a custom coil designed to receive signals in the approximate range of 9-315 kHz, which is the allowed bandwidth for active medical implant systems defined by the European Radiocommunications Committee 70-03. The coil was placed in an airtight plastic sleeve and submerged in close proximity to the cables and pads. The measured signals were monitored on the oscilloscope. The function of the PG was evaluated using the PRM via the wanded telemetry link.

\section{Methods}

Wanded communication with the PG was established prior to powering $\mathrm{ON}$ the defibrillator. The integrity of the wanded link between the PG and PRM was then verified after powering ON the defibrillator. Wanded communication remained stable without any interruption observed on the PRM real-time telemetry display.

Next, the pacing function of the defibrillator was switched to the "standby" position, and an attempt to establish wanded communication between the PG and PRM was repeated. This time, significant noise and numerous dropouts in the wanded communication were observed on the PRM real-time telemetry display. The signal degradation when the defibrillator was switched to "standby" made it impossible to maintain wanded communication between the PG and PRM.

The wanded communication session between the PRM and PG was then closed and the PRM was then powered OFF. With only the defibrillator powered ON, spurious radiated emissions (signals) from the cables and pads were captured using the custom coil and analyzed on the oscilloscope. The combined intentional and spurious (unintentional) emissions produced by the defibrillator resulted in complex waveforms, which were measured with the custom receive coil. The recorded oscilloscope waveforms are provided in Figures 3 and 4, showing the amount of EMI when the defibrillator pacer "standby" function was in the $\mathrm{ON}$ and $\mathrm{OFF}$ positions.

\section{Discussion}

Signal-to-noise (SNR) ratio compares the level of the desired signal (programmer or implantable telemetry signal) to the level of environmental noise (e.g., constant current noise or other background noise produced by the defibrillator). ${ }^{4}$ 

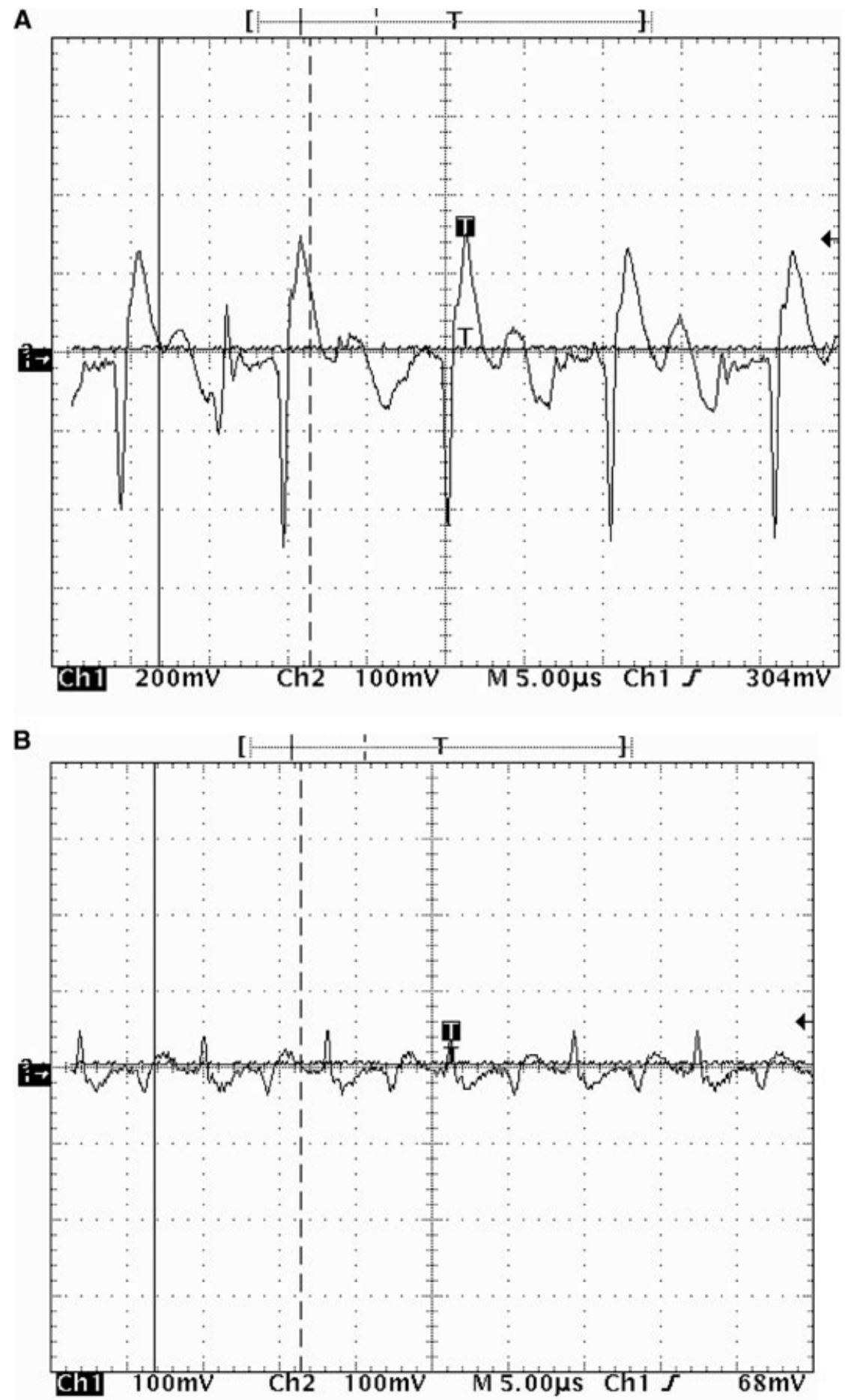

Figure 3. External defibrillator. Philips Heartstart XL, M4735A. (A) EMI detected by the oscilloscope with pacer in "standby" position (scale factor $=200 \mathrm{mV}$ per division). (B) EMI detected by the oscilloscope with pacer in OFF position (scale factor $=100 \mathrm{mV}$ per division).

A loss of wanded telemetry is possible if environmental noise is sufficient to reduce the SNR below the level required to properly demodulate the telemetry link data transmitted to or from the pacemaker. Boston Scientific bench testing indicates the pads and cables of the defibrillators under investigation generate sufficient environmental noise to cause EMI with the 

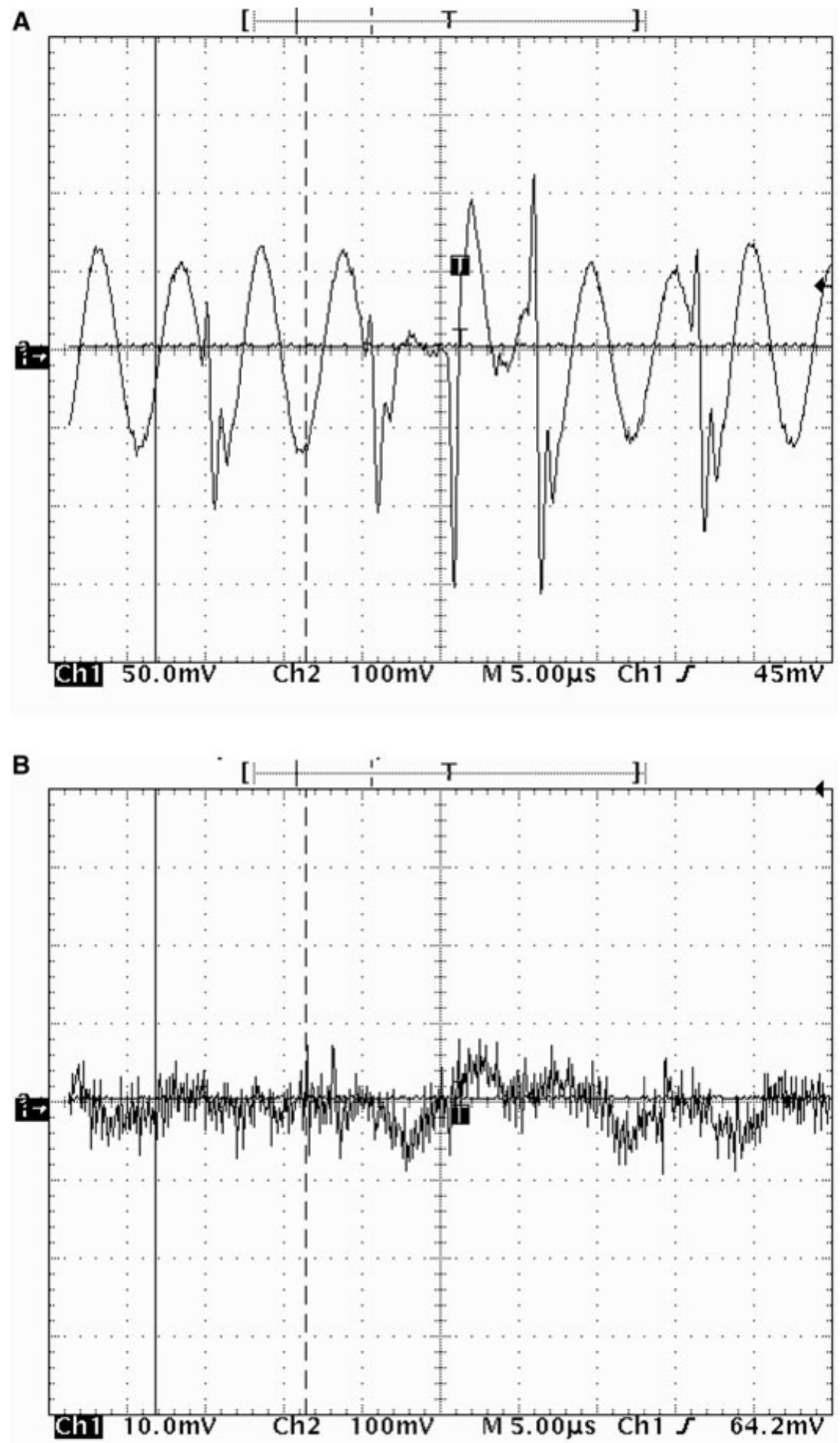

Figure 4. External defibrillator. Hewllet-Packard Codemaster, M1722B. (A) EMI detected by the oscilloscope with pacer in "standby" position (scale factor $=50 \mathrm{mV}$ per division). (B) EMI detected by the oscilloscope with pacer in OFF position (scale factor $=10 \mathrm{mV}$ per division). 
wanded communication link. Specifically, the radiated noise from the pads and cables exists in the same frequency band required to operate the wanded communication link $(\sim 9-315 \mathrm{kHz})$. Boston Scientific implantable devices operate in the range of 50-150 kHz; however, exact operating frequencies are proprietary.

It is theorized that an impedance measurement scheme is employed by the defibrillators, which utilizes a high-frequency current. This inband noise causes an increase in the noise floor, making it more difficult to maintain a robust link. The presence of an increased noise floor is supported by the relative defibrillator signal measurements shown in Figures 3 and 4. Recognizing the specified scaling factors on the plots, an increase greater than $7 \times$ was observed when the Philips Heartstart Pacer standby function was switched ON ( $\sim 760 \mathrm{mV}$ peak to peak) compared to when it was switched OFF $(\sim 100 \mathrm{mV})$. Similarly, note the increase by more than $16 \times$ when the Hewlett-Packard Codemaster Pacer standby function was switched ON $(\sim 270 \mathrm{mV})$ compared to when it was switched OFF ( $\sim 16 \mathrm{mV})$. All oscilloscope measurements were made within the specified in-band range of $9-315 \mathrm{kHz}$.

The power of the wanded signal from the PG is less than one-tenth that of the PRM; therefore, the PRM may be unable to maintain wanded communication because it is unable to distinguish between the in-band noise created by the defibrillator pads/cables and the response from the PG. In the absence of the environmental noise, wanded communication is possible at a distance of approximately $5 \mathrm{~cm}$. In the presence of sufficient in-band environmental noise, the distance required to maintain a wanded communication link may be reduced. Powering OFF the defibrillator will allow the wanded communication link to return to its normal function. The result of the described interference has the same effect as increasing the distance of the telemetry programming wand beyond the specified distance required to maintain communication with the PG.

\section{References}

1. Pinski SL, Trohman RG. Interference in implanted cardiac devices, Part I. Pacing Clin Electrophysiol 2002; 25:1367-1381.

2. Pinski SL. Electromagnetic Interference and Implantable Devices. In: Ellenbogen KA, Kay GN, Lau CL, and Wilkoff BL (eds.) Clinical Cardiac Pacing, Defibrillation, and Resynchronization Therapy. Philadelphia, PA, Saunders: 2007, pp. 1150-1176.

3. Association for the Advancement of Medical Instrumentation. Active
Evaluation of intracardiac electrograms stored in PG memory confirmed that the PG maintained its programmed therapy function during exposure to the environmental noise produced by the defibrillator in its various operating modes.

\section{Limitations}

This EMI interaction is documented only between the two defibrillators available at our institution and Boston Scientific devices. We are unable to comment about the other defibrillators and heart rhythm devices of other manufacturers. The in vitro experiment was limited to confirm the clinical observations. Also, there is no standardized in vitro torso simulator. The experiments were carried out with the guidance of the AAMI PC69: 2007 recommendations for an in vitro torso simulator. ${ }^{3}$ Finally, the in vitro experiment utilized a saline bath with the implanted device between the defibrillation pads. The saline and defibrillation pad positioning does not exactly represent the skin, fat, muscle, bone, and blood in our patients. The saline provides low impedance and may constitute a more severe electrical model than that found in clinical practice; however, the EMI was controlled by the position of the external pacer mode for both in vivo and in vitro, suggesting that the saline bath was adequate to reproduce the phenomenon.

\section{Conclusions}

We documented that EMI was produced when the pacing mode on the defibrillator was in the "standby" position, and we discovered that by switching the pacing mode to the OFF position, the CRT-P device could be interrogated without difficulty. This is the first case series reporting EMI with Boston Scientific CRT-P Contact Renewal device H120/H125 wanded telemetry from the defibrillator with pacing mode on standby. The CRT-P device maintained its programmed therapy function throughout exposure to the environmental noise produced by the defibrillator. Failure to recognize this potential interaction may lead to inappropriate device and resource utilization.

implantable medical devices - Electromagnetic compatibility - EMC test protocols for implantable cardiac pacemakers and implantable cardioverter defibrillators. ANSI/AAMI PC69:2007. Available at: www.aami.org (accessed May 1, 2010).

4. Pinski SL, Trohman RG. Interference in implanted cardiac devices, Part II. Pacing Clin Electrophysiol 2002; 25:14961509. 